\title{
Effect of Equivalence Ratio and Turbulence Fluctuations on the Propagation of Detonations
}

\author{
Damien Masselot*, Romain Fiévet ${ }^{\dagger}$ and Venkat Raman ${ }^{\ddagger}$ \\ Department of Aerospace Engineering, University of Michigan, Ann Arbor, MI 48109-2102, USA
}

The effect of turbulence and inhomogeneities on the propagation of a 3-dimensional detonation is studied in a practical configuration. An in-house Navier-Stokes solver using detailed $\mathrm{H}_{2}-\mathrm{O}_{2}$ chemistry is first validated on known test-cases, then used on the target calculation. The geometry chosen is that of a linearized RDE with non premixed injection, operating at low initial pressure and temperature. The results are analyzed with an emphasis on two important metrics describing the nature of the detonation, shock front velocity and induction length.

\section{Introduction}

Rotating detonation engines (RDEs) form a class of pressure-gain combustion systems that can augment the efficiency of conventional gas turbine operations. In such systems, a continuous detonation wave propagates through a fuel/air mixture to release heat and increase pressure in a constant volume combustor. RDEs are considered operationally superior to pulse detonation engines due to the continuous pressure gain that reduces the complexity of coupling with downstream turbine sections. Nevertheless, developing a robust design where such detonations can be sustained is a formidable challenge.

The main issue is in the design of an injection system that can deliver fresh fuel/air mixture while not affected by the passing detonation wave. In preliminary designs, fuel and air were mixed upstream and fed into the combustion chamber normal to the direction of the detonation wave propagation. If a simple jet is used for injection, the pressure rise as the detonation wave passes the injection hole will stop mass flow, which can be overcome by increasing the injection pressure. However, this reduces the efficiency obtained through pressure gain combustion. Further, the detonation wave can pass through the injector and can ignite the mixture in the mixing tube, which can lead to catastrophic failure. In general, some level of non-premixed injection is desirable, as this will have the effect of dissipating the detonation wave as it gets closer to the injection port. The design challenge is to determine the impact of such non-premixing on the stability and propagation of a detonation wave.

Most of the existing literature concerning detonation of hydrocarbon or hydrogen fuels focus on propagation in a uniform premixed fuel/air mixture. A number of studies have tested the propagation speed (C-J velocity, for instance) as a function of composition, pressure, temperature and other operating conditions. ${ }^{1-5}$ A similar study of spatially non-uniform mixtures is rather limited. Kessler et al. ${ }^{6}$, Ettner et $a l .{ }^{7}$ and Calhoon and Sinha ${ }^{8}$ have studied numerically the impact of a $1 \mathrm{D}$ transverse concentration gradient on cellular detonation patterns and the shape of the detonation front in a 2 dimensional channel. Calhoon and Sinha ${ }^{8}$ found that a large concentration gradient led to a decrease in detonation velocity $(\approx 6 \%)$ ) and created a parabolic detonation front before quenching it. In their study, Kessler et al. ${ }^{6}$ investigated the influence of the gradient intensity, channel width, and reactant activation energy on the velocity, cell structure and quenching of the detonation. Ettner et al. ${ }^{7}$ found to what extent gradient intensity impacted the detonation front curvature and could sometimes cause Mach reflection in the channel, and compared

${ }^{*} \mathrm{PhD}$ Student, AIAA Student Member.

${ }^{\dagger}$ PhD Student, AIAA Student Member.

¥Associate Professor, AIAA Associate Fellow.

Copyright (C) 2017 by Damien Masselot. Published by the American Institute of Aeronautics and Astronautics, Inc. with permission. 
the results obtained when using single step or detailed chemistry. Those simulations were compared to the experimental work of Ishii and Kojima ${ }^{9}$, which treated of similar configurations. Additionally, Jin et al. ${ }^{10}$ and Massa et al. ${ }^{11}$ have investigated interactions between turbulence and 3-dimensional detonation, using a 1-step chemistry model. Massa et al. ${ }^{11}$ compared the effect of turbulence on velocity profiles of a detonation wave in both a non-reacting case and a 1-step chemistry approximation. Jin et al. ${ }^{10}$ studied how different types of turbulence impacted the detonation cellular structure and post detonation turbulence. Finally Fujii et al. ${ }^{12}$ have studied the impact of premixed and non-premixed injection on the average detonation velocity in a $2 \mathrm{D}$ case, using detailed chemistry.

A second important physical process to consider is the turbulence near the injection port. Due to the high injection speeds, as well as the presence of the fast-moving detonation wave, both baroclinic forces and regular inertial instabilities will cause the formation of turbulence structures. RDEs typically use an annular design, where a thin annular region forms the main combustion domain. Due to the nearly two-dimensional nature of such systems, the development of turbulence is vastly different from conventional three-dimensional turbulence found in other gas turbine applications. While numerical studies have shown that both threedimensional Navier-Stokes and two-dimensional Euler simulations yield similar solutions, ${ }^{13}$ their effect on the homogenization (mixing) of non-uniform fuel-air mixtures is not well understood.

With this background, the present work investigates the effects inhomogeneities in the reactant mixture have on the propagation and structure of a detonation wave. For this purpose, discrete fuel injectors replicating emerging RDE designs are used. Using detailed numerical simulations that capture the chemical kinetics as well as the shock structure, the effect of such fuel injection on wave propagation is studied. In particular, the impact of equivalence ratio variations on the induction length for fuel-air mixture to ignite is studied. The low pressure detonation of a highly diluted $\mathrm{H}_{2}-\mathrm{O}_{2}-\mathrm{Ar}$ mixture at $300 \mathrm{~K}$ has been chosen as the main focus of this study, as it has been used in a number of other works to understand the stability and regularity of the cellular detonation pattern it creates.

The paper is organized as follows: Section II. discusses the computational solver used and provides related numerical details, Section III. focuses on solver validation using two well known problems (1D and 2D) and finally Section IV. contains a description and analysis of the results obtained for the 3 dimensional non-premixed simulation.

\section{Computational and simulation details}

A direct numerical simulation approach is used to study detonation propagation in non-premixed mixtures. For this purpose, simulations have been performed using the in-house compressible flow solver, UTComp, that has already been used for a number of supersonic reacting flow problems by the mean of both Large Eddy Simulations and Direct Numerical Simulations. ${ }^{14-18}$ The governing equations of fluid flow for the detonation problem are given by

$$
\frac{\partial \rho}{\partial t}+\frac{\partial \rho u_{j}}{\partial x_{j}}=0
$$

and

$$
\frac{\partial \rho u_{i}}{\partial t}+\frac{\partial \rho u_{j} u_{i}}{\partial x_{j}}=-\frac{\partial P}{\partial x_{i}}+\frac{\partial \tau_{i j}}{\partial x_{j}},
$$

where $\rho$ is the fluid density, $u_{i}$ is the velocity vector, $P$ is the pressure and $\tau_{i j}$ is the Newtonian stress tensor. The mass-fraction of each species is determined using the transport equation:

$$
\frac{\partial \rho Y_{\alpha}}{\partial t}+\frac{\partial \rho u_{j} Y_{\alpha}}{\partial x_{j}}=\frac{\partial}{\partial x_{j}} \rho D \frac{\partial Y_{\alpha}}{\partial x_{j}}+\rho S_{\alpha}(Y)
$$

where $Y=\left[Y_{1}, \cdots, Y_{N-1}\right]^{T}$ is the vector of $N-1$ species mass fractions and $S_{\alpha}$ the reaction source term. Argon is the $N$-th species and its mass fraction is obtained as $1-\sum_{\alpha=1}^{N} Y_{\alpha}$. The transport equation for the total energy of the system writes :

$$
\frac{\partial \rho e_{t}}{\partial t}+\frac{\partial u_{j}\left(\rho e_{t}+P\right)}{\partial x_{j}}=\frac{\partial}{\partial x_{j}} k \frac{\partial T}{\partial x_{j}}-\frac{\partial}{\partial x_{j}}\left(\tau_{i j} u_{i}\right)+Q_{r e a c}
$$

where $Q_{\text {reac }}$ is the energy released by chemical reactions. 
A 9-species 19-reaction chemical mechanism for hydrogen-air combustion is used, ${ }^{19}$ and implemented using CHEMKIN ${ }^{20}$ based subroutines. Transport properties and heat capacity are computed using NASA polynomial fits. ${ }^{21}$

The solver is designed for structured grids, and uses a fifth-order WENO scheme ${ }^{22}$ for computing the non-linear convective fluxes. The nonlinear scalar terms are computed using a bounded QUICK scheme. ${ }^{23}$ Viscous and diffusion terms are computed using a 4-th order central scheme. The compressible solver uses cell-centered collocated variable arrangement, and explicit time-stepping for this flow configuration. A 4-th order Runge-Kutta scheme is used for temporal discretization. The code is parallelized using MPI-base domain decomposition, and has been shown to scale up to $10 \mathrm{~K}$ cores linearly for problem sizes similar to those considered in this work.

\section{Validation of solver}

Although the UT-COMP solver has been used extensively for shock-containing problems, this is the first application to detonation conditions. In order to ensure that the numerics do not adversely affect results, verification and validation studies are conducted.

\section{A. One-dimensional detonation case}

The 1-D detonation case has been solved using a highly resolved detailed chemistry solver, developed independent of UT-COMP and based on the method of Powers and Paolucci. ${ }^{24}$ Simultaneously, the UT-COMP solver was adapted for 1-D configuration. The verification problem consists of a 1-dimensional detonation wave in a stoichiometric $\mathrm{H}_{2}-\mathrm{O}_{2}$ mixture diluted in $70 \%$ Argon (mole fraction) at $\mathrm{T}_{0}=298 \mathrm{~K}$ and $\mathrm{P}_{0}=6670 \mathrm{~Pa}$. A 1-dimensional uniform domain $(\Delta x=50 \mu \mathrm{m})$ was initialized as an unburnt mixture and then perturbed on one side with a strong blast wave. This established a detonation wave after a transition period, resulting the well-studied Zel'dovich-von Neumann-Döring (ZND) detonation structure. The Chapman-Jouguet velocity for this mixture is $1618 \mathrm{~m} / \mathrm{s}$ (obtained from the analytical 1-D solution), while the UT-COMP simulations yielded a velocity of $1608 \mathrm{~m} / \mathrm{s}$. Figure 1 illustrates the comparison of the detonation structure between the two solvers, and shows very good agreement. This establishes basic verification of the UT-COMP detonation solution capability.

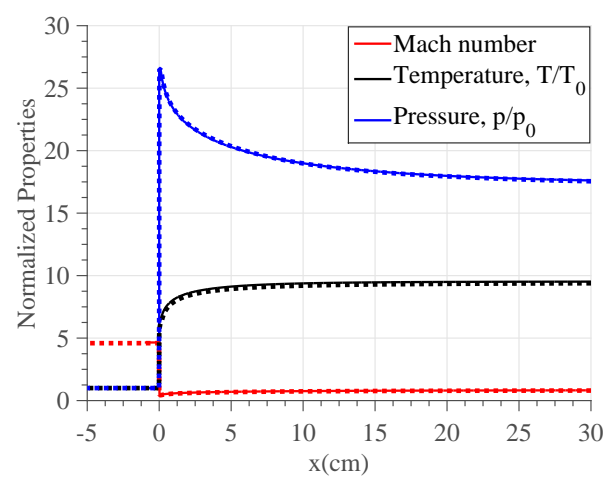

Figure 1. 1D detonation properties (solid line : exact - dotted : UTComp)

\section{B. Two-dimensional detonation case}

A standard test case for detonations is the propagation of a perturbed detonation wave in a confined twodimensional channel. The perturbation gives rise to a transverse instability, the generation of triple points, and the formation of cell structures (observed on smoke-foil plots). The size of the cells is an important indicator of the propensity of the fuel mixture to detonate, with smaller cell sizes implying higher detonability.

A 2-D detonation wave based on the configuration of Oran et al. ${ }^{2}$ is computed. The domain is discretized using a uniform orthogonal grid system consisting of $\left(N_{x}, N_{y}\right)=(4000,256)$ points, and spans a distance of $60 \mathrm{~cm}$ in a $6 \mathrm{~cm}$ high channel. The simulation is initialized using a 1-D detonation profile near the left boundary, along with a patch of different species profiles to emulate a perturbation in the flow. The cellular 
structures are regular after an initial transition period, at which point, the flow is translated back to the left boundary and continues to develop. This procedure allows an essentially infinite-length channel.

The first plot of Figure 2 shows the pressure contours after the detonation wave has had some time to develop into a system of transverse shockwaves and 4 triple points. The detonation's average speed is found to be the same as the 1D propagation speed. To best way to observe the "cellular" nature of the detonation, it is interesting to look at the map of the maximum local heat release history (Figure 2), which essentially gives the position history of each triple point. Detonation cell sizes can be extracted from this data, which gives us an an average of approximately $5.7 \times 3 \mathrm{~cm}$, which is in good agreement with numerical data ${ }^{2}$ found in literature for this mixture.

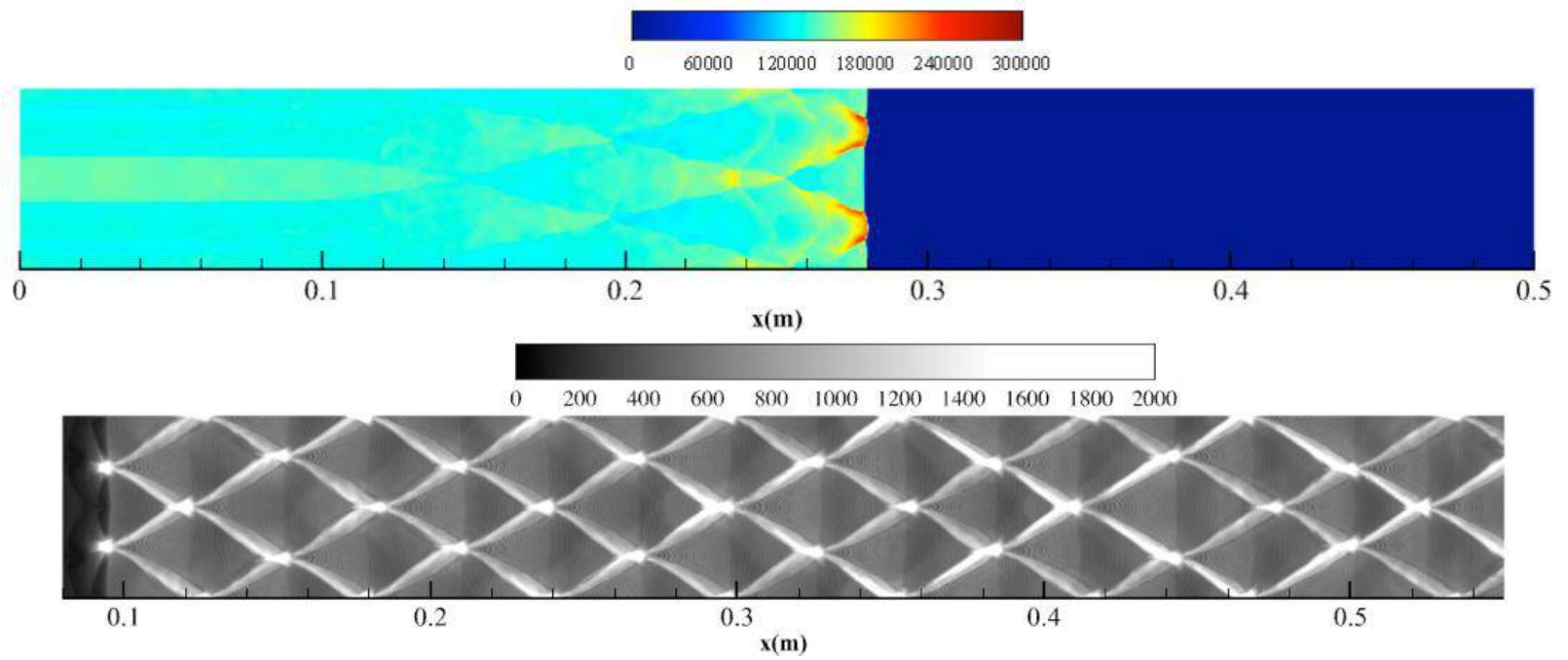

Figure 2. 2D channel detonation - Top: Pressure contours (Pa) - Bottom: Maximum heat release history ( $\mathbf{J} / \mathbf{m}^{3}$ )

In such transverse detonations, two additional quantities of interest are the shock-front propagation velocity and the induction length. In particular, the spatial variation of these quantities is useful to study, due to their relation to a non-premixed injection configuration.

Analyzing the shock front speed gives information as to how the detonation wave gets distorted as it propagates through the channel. The shock front can be characterized by the sharp increase in pressure following the wave, which allows to determine the exact location of the discontinuity, as pressure is uniform in the unreacted part of the domain. The shock front velocity in the propagation direction can be extracted using the shock position data at each spanwise location, resulting in a curved 1-D profile at each time step.

The induction length is defined here as the distance between the shock front and the beginning of the reaction zone in the propagation direction. The reaction front has been characterized as the closest position to the shock front where the mass fraction of oxygen atoms reaches a certain threshold. Note that a number of other species might have been used to that end and this choice is relatively arbitrary, but very similar results are found when using other species. It is relevant to study induction length to compare premixed and non-premixed detonation. Indeed, a number of detonation simulations of $2 \mathrm{D}^{25-27}$ and $3 \mathrm{D}^{28}$ systems in literature use an induction-time parameter model (IPM) to compute chemical source terms. This method has proved to be accurate for premixed systems, but extension of IPM to non-premixed systems might be non-trivial. ${ }^{29}$

Figures 3 and 4 are contour plots of the shock front velocity and the induction length, respectively, as a function of the local shock front position. These results show a behaviour that is in agreement with theoretical analysis of the cellular detonation structure. When two triple points collide, or a triple point bounces against a wall, it generates a "burst" that locally accelerates and curves the shock front. The velocity then slowly decays until a new collision occurs. As far as induction length is concerned, the collisions reattach the reaction front to the shock front, and the induction length increases throughout the detonation cell, i.e. the reaction zone detaches from the shock front until a new collision occurs. Hence, when such transverse detonations are observed, induction length and shock-velocity variations are significant. In the next section, 
similar analysis for non-premixed injection will be conducted.

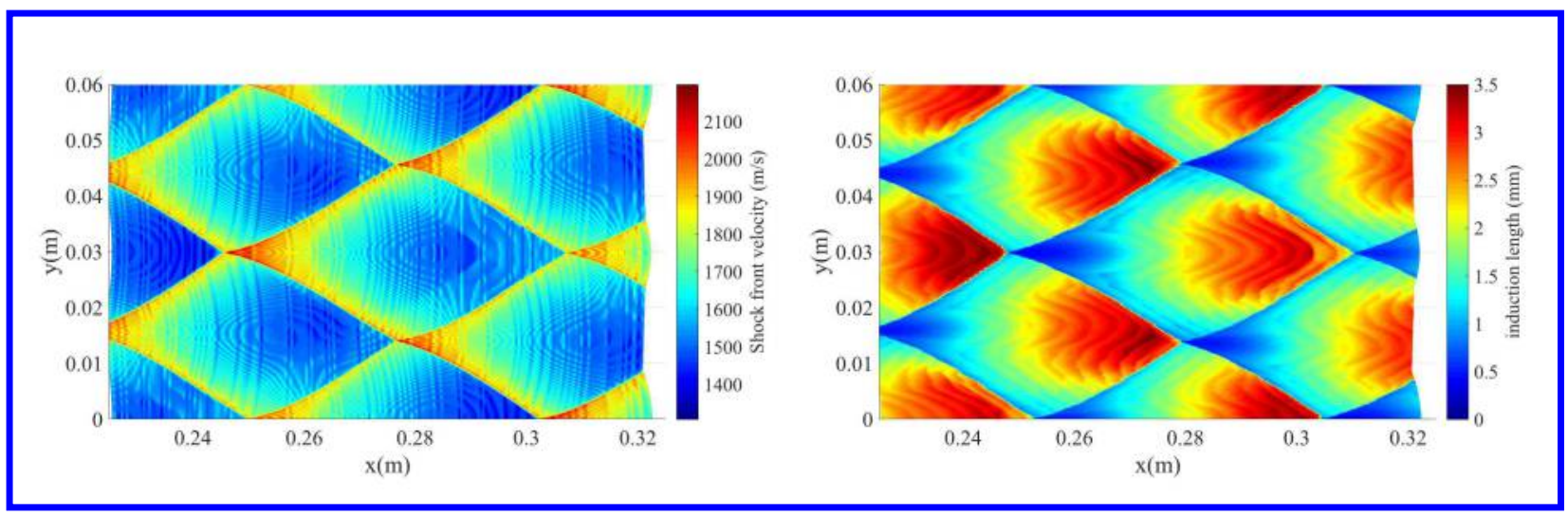

Figure 3. Shock front velocity in premixed channel

Figure 4. Induction length in premixed channel

\section{Detonation propagation in non-premixed turbulent flows}

\section{A. Simulation configuration}

The main simulation geometry is meant to recreate the injection region of a "linearized" RDE with nonpremixed injection, where only the fuel injection would be considered. It consists in a 3-D rectangular region of dimensions $15 \mathrm{~cm} \times 6 \mathrm{~cm} \times 2 \mathrm{~cm}$, filled with an ambient mixture of $\mathrm{O}_{2}-\mathrm{Ar}(1: 7$ in moles) at $298 \mathrm{~K}$ and $6670 \mathrm{~Pa}$. The ratio of $\mathrm{O}_{2}$ to Ar concentration is constant between this simulation and the premixed channel simulation, only $\mathrm{H}_{2}$ is not present in this mixture. Pure $\mathrm{H}_{2}$ is supplied from the bottom wall through a series of 16 cylindrical injectors (2mm diameter, $6.6 \mathrm{~mm}$ spacing between centers) at $298 \mathrm{~K}, 6670 \mathrm{~Pa}$ and at a mach number approaching the choked condition $(\mathrm{Ma}=0.83)$.

The top and right boundaries are set as outflows, while the domain is periodic in the spanwise direction. The left boundary serves as an inflow. This inflow condition is used to introduce a right-running detonation wave into the domain, which was generated using the outflow of a 3D stoichiometric premixed channel simulation analogous to the $2 \mathrm{D}$ example presented above.

The grid used for this simulation is uniform with $\Delta \mathrm{x}=0.15 \mathrm{~mm}, \Delta \mathrm{z}=0.133 \mathrm{~mm}$ everywhere, and

$$
\Delta y=\left\{\begin{array}{l}
0.133 \mathrm{~mm} \text { if } \mathrm{y}<4 \mathrm{~cm} \\
0.2 \mathrm{~mm} \text { if } \mathrm{y}>4 \mathrm{~cm}
\end{array}\right.
$$

In the first part of the simulation, the detonation wave is not yet present and the jets are given time to develop and start breaking down into smaller structures. After that initialization, the chamber is partially filled with $\mathrm{H}_{2}$ from the injectors, as can be seen on Figure 5, and the detonation starts propagating from the inflow.

\section{B. Simulation Results}

The results from the non-premixed simulation are presented at different times in Figure 6 . Here, the $Y_{\mathrm{H}_{2}}$ isosurface is used to locate the fuel jets, the $Y_{O H}$ locates the reaction zone and the T isosuface gives the shock location. The first two snapshots show reaction species from the premixed channel being convected into the domain, lagging behind the shock. After the shock crosses the first injector, a new reaction front appears in the lower part of the domain, while no reaction occurs in the upper zone where no fuel is present. The shock front is curved in the lower part of the domain due to the local acceleration induced by the presence of $\mathrm{H}_{2}$ lowering the density. Conversely, the shock front tends to flatten in the upper part of the domain where the initial mixture is homogeneous.

The shock front velocity and induction length are computed in the center plane along the spanwise direction $\mathrm{z}$ for this non-premixed simulation. The shock front velocity is first considered, as shown in Figure 7. Figure 8 shows the result of the averaging of the shock front speed with respect to $\mathrm{x}$. It is seen that the shock front velocity is highly correlated to the injector locations. This is mainly due to the large density 


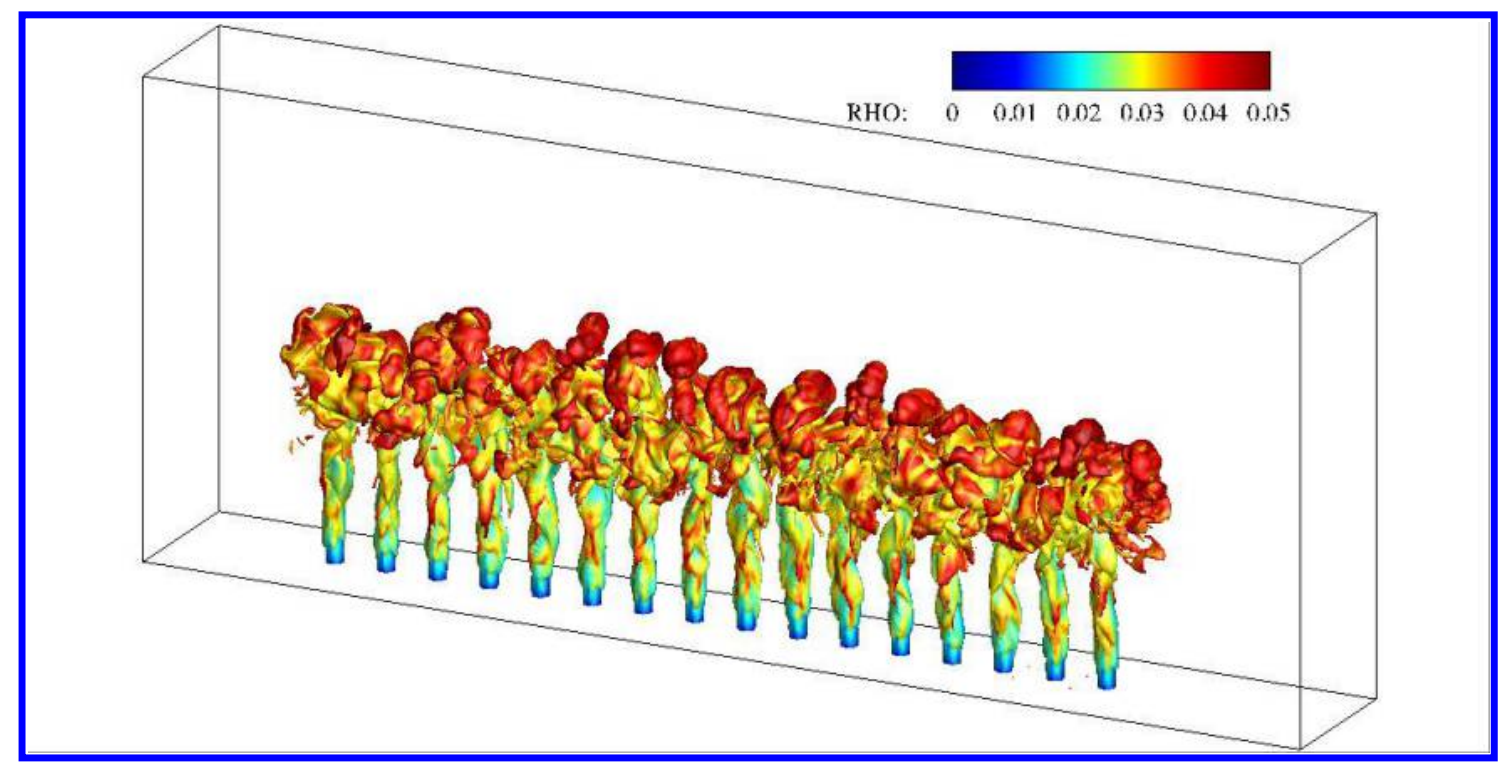

Figure 5. Isosurface of $Y_{H_{2}}=0.15$, colored by density

drop resulting from the high concentration of $\mathrm{H} 2$ directly above the jets, which locally accelerates the shock wave component of the detonation. In the most turbulent region, the correlation is slightly less important, as the density inhomogeneities start dissipating. Note that the velocity is in some regions greater than twice that of the Chapman-Jouguet detonation for the stoichiometric mixture. All those observations suggest that the shock front velocity quickly adapts to density and mixture variations. It is also interesting to notice that there is no cellular detonation structure to be observed, although there exists some triple points that propagate through the upper non-detonable region.

The induction length pattern for this configuration (Figure 9) also presents interesting characteristics. The domain can be divided into three zones. The upper region $(\mathrm{y} \in[0.04,0.06])$ and the left and right regions $(\mathrm{x} \in[0,0.015] \cup[0.125,0.14])$ correspond to the first zone, where very little $\mathrm{H}_{2}$ is present and therefore the detonation cannot be sustained. In each of those regions, the reaction front lags behind the shock front, as it is only being convected to the right and not being fed any fuel. This can be observed on Figure 9, especially in the upper region, where the shock front fully decouples from the reaction zone.

The second zone is the lower part of the jets $(\mathrm{y} \in[0,0.02])$ where there is very little break up and turbulence intensity is fairly low. In this region, the reaction front reattaches shortly after the wave hits the first jet, and stays attached until it crosses the last jet. There also is an apparent correlation between induction length and injector location, i.e. the induction length oscillates around its theoretical ZND value.

The third zone is the upper part of the jets $\left(y \in\left[\begin{array}{ll}0.02 & 0.04\end{array}\right]\right)$ where they are breaking down into small structures and turbulence dominates the flow. There, the reaction front only sporadically reattaches to the shock front, and can lag very far behind it, leading to an induction length up to 5 times larger than the ZND value and higher on average than in premixed systems. The initial reattachment of the reaction zone is also significantly delayed compared to the lower zone. There is no observable correlation between induction length and injector location in this region. These observations hint to the fact that turbulence affects induction time, and should be taken into account when attempting to model combustion using IPM. However, these results alone are not sufficient to fully quantify the impact of turbulence on induction time, as other factors might have played a role in the final results (species mass fraction and density gradients).

\section{Conclusions}

A three dimensional simulation of a 3-D linearized RDE configuration with non-premixed injection has been carried out. The impact of the turbulence generated by the presence of fuel injectors has been characterized by considering the shock velocity and induction length of the detonation wave. It has been found that the shock front velocity is sensitive to density and mixture variations but not greatly affected by the turbulence intensity in the flow. Conversely, it has been found that induction length is greatly increased in turbulent regions of the flow, but slower to adapt to mixture and density variations. 


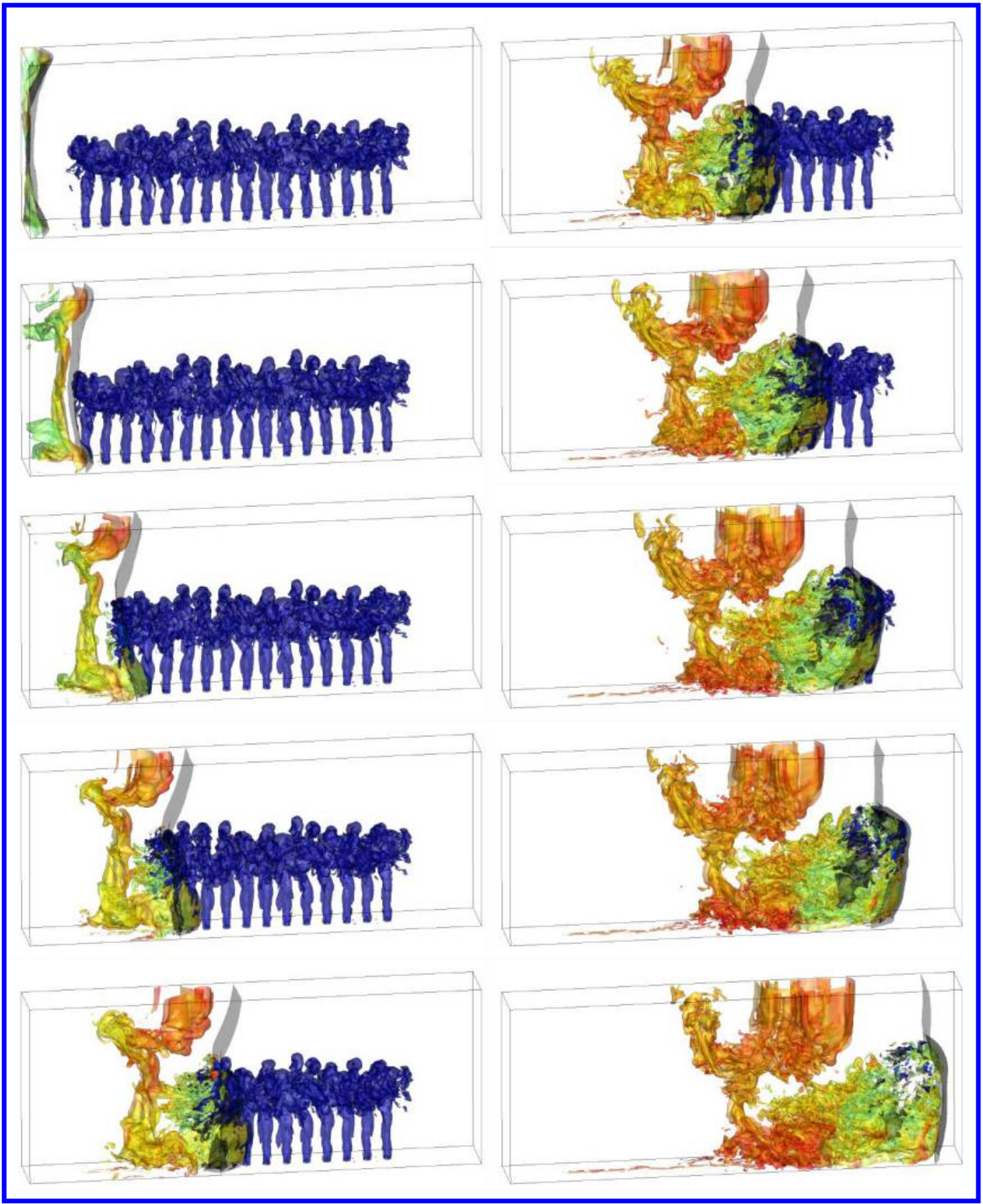

Figure 6. Time evolution of detonation wave - Isosurface of $Y_{H_{2}}=0.12$ and $Y_{O H}=0.006$

\section{Acknowledgements}

This research is supported by DOE-NETL grant DE-FE0025315 with Dr. David Lyons as program monitor. The authors also thank NASA HECC and Texas Advanced Computing Center for generous allocation 

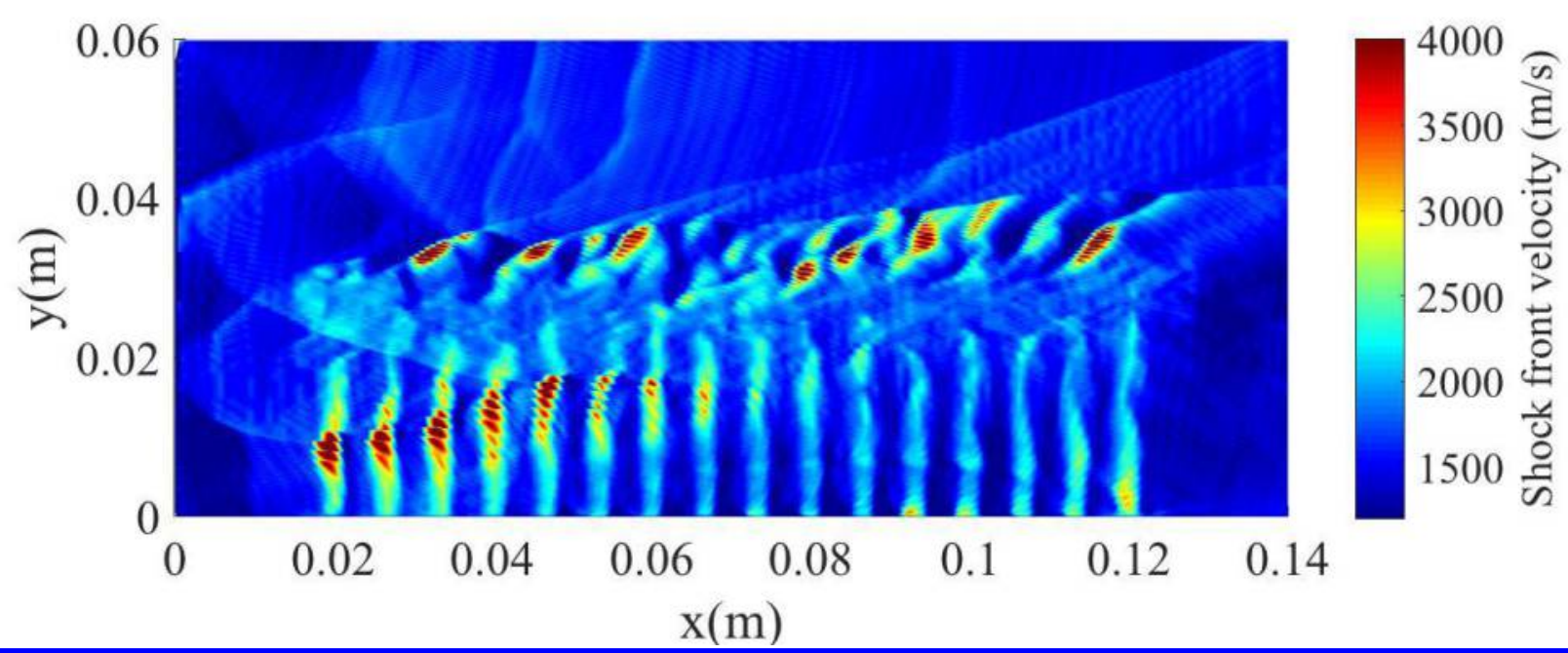

Figure 7. Shock front velocity in the $\mathrm{z}$ center plane

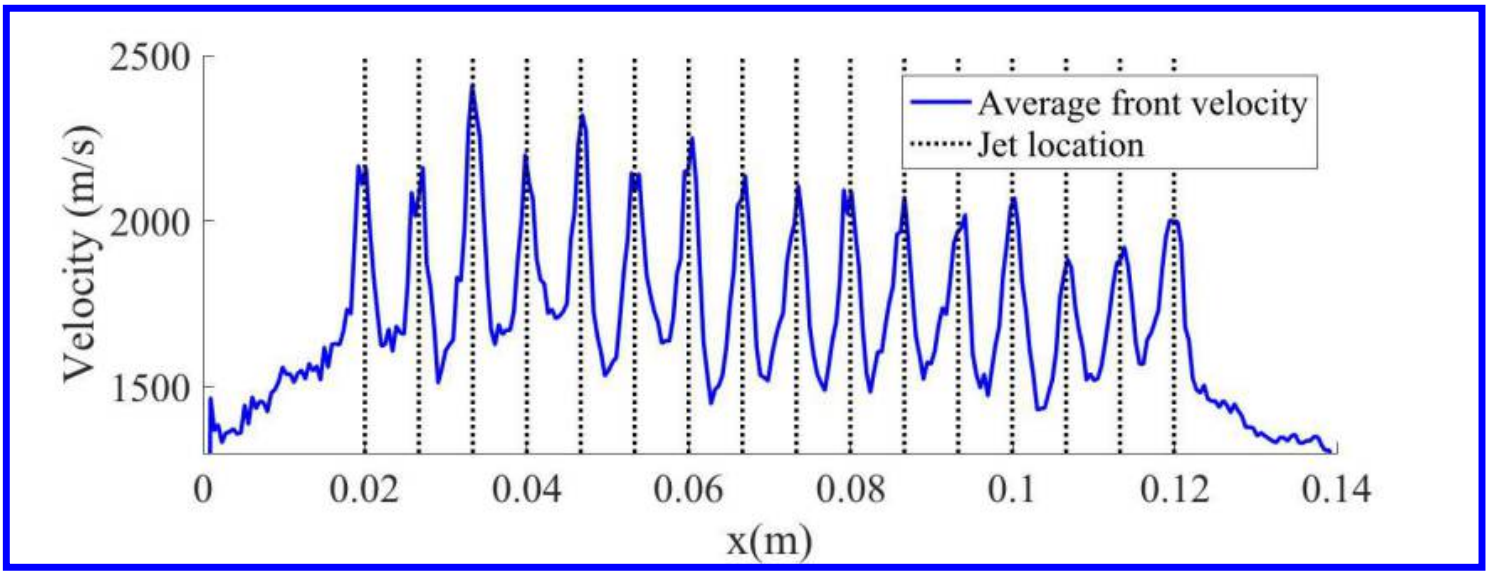

Figure 8. Average Shock front velocity in detonation region of the center plane

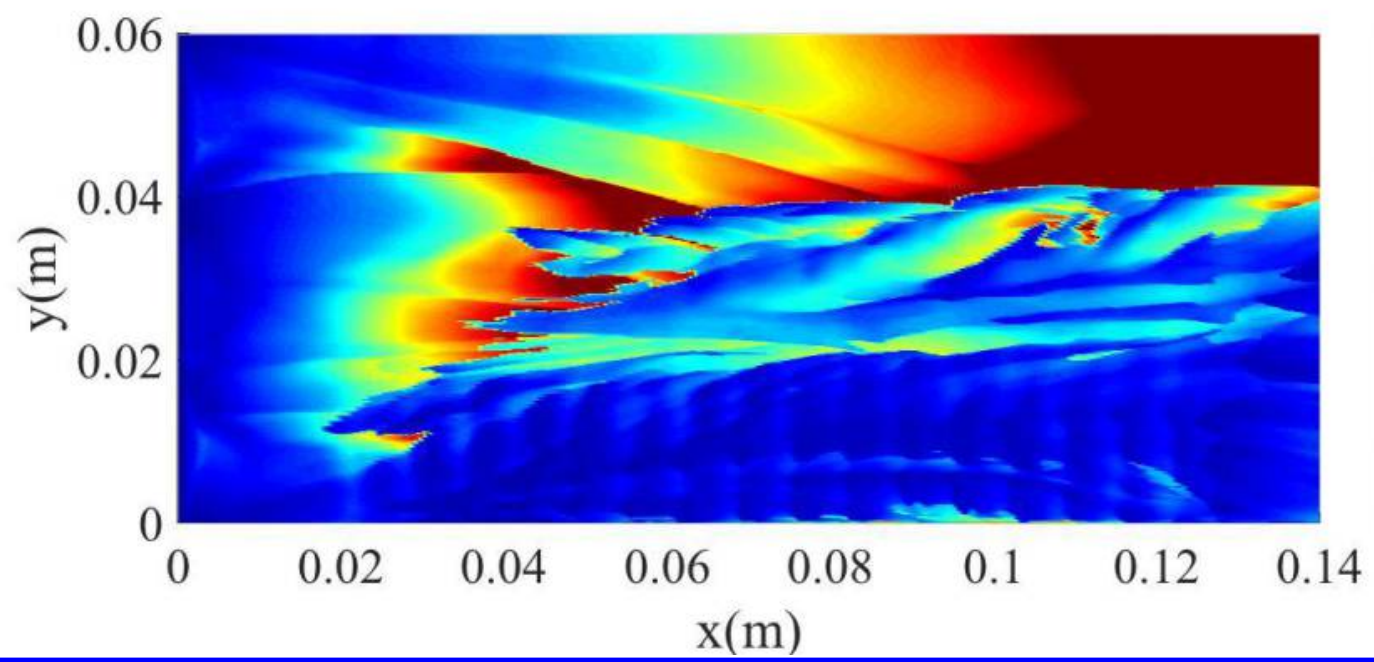

Figure 9. Induction length in the $\mathrm{z}$ center plane

of computing time. 


\section{References}

${ }^{1}$ Shepherd, J. E., "Detonation in gases," Proceedings of the Combustion Institute, Vol. 32, No. 1, 2009, pp. 83-98.

${ }^{2}$ Oran, E. S., Jr., J. W. W., Stefaniw, E. I., Lefebvre, M. H., and Jr., J. D. A., "A Numerical Study of a Two-Dimensional H2-O2-Ar Detonation Using a Detailed Chemical Reaction Model," Combustion and Flame, Vol. 113, No. 12, 1998, pp. 147 163.

${ }^{3}$ Kailasanath, K., Oran, E., Boris, J., and Young, T., "Determination of detonation cell size and the role of transverse waves in two-dimensional detonations," Combustion and Flame, Vol. 61, No. 3, 1985, pp. 199 - 209.

${ }^{4}$ Inaba, K., Matsuo, A., and Tanaka, K., "Numerical Investigation on Acoustic Coupling of Transverse Waves in TwoDimensional $\mathrm{H}_{2}-\mathrm{O}_{2}$-Diluent Detonations," Japan Society of Aeronautical Space Sciences Transactions, Vol. 47, 2005, pp. 249255.

5 Taylor, B., Kessler, D., Gamezo, V., and Oran, E., "Numerical simulations of hydrogen detonations with detailed chemical kinetics," Proceedings of the Combustion Institute, Vol. 34, No. 2, 2013, pp. 2009 - 2016.

${ }^{6}$ Kessler, D. A., Gamezo, V. N., and Oran, E. S., "Gas-phase detonation propagation in mixture composition gradients," Philosophical Transactions of the Royal Society A - Mathematical Physical and Engineering Sciences, Vol. 370, No. 1960, SI, FEB 13 2012, pp. 567-596.

${ }^{7}$ Ettner, F., Vollmer, K. G., and Sattelmayer, T., "Mach reflection in detonations propagating through a gas with a concentration gradient," Shock Waves, Vol. 23, No. 3, MAY 2013, pp. 201-206.

${ }^{8}$ Calhoon, W. and Sinha, N., Detonation Wave Propagation in Concentration Gradients, American Institute of Aeronautics and Astronautics, 2005.

-9 Ishii, K. and Kojima, M., "Behavior of detonation propagation in mixtures with concentration gradients," Shock Waves, Vol. 17, No. 1-2, AUG 2007, pp. 95-102.

${ }^{10}$ Jin, T., Luo, K., Dai, Q., and Fan, J., "Simulations of Cellular Detonation Interaction with Turbulent Flows," AIAA JOURNAL, Vol. 54, No. 2, FEB 2016, pp. 419-433.

11 Massa, L., Chauhan, M., and Lu, F. K., "Detonation-turbulence interaction," Combustion and Flame, Vol. 158, No. 9, SEP 2011, pp. 1788-1806.

12Fujii, J., Kumazawa, Y., Matsuo, A., Nakagami, S., Matsuoka, K., and Kasahara, J., "Numerical investigation on detonation velocity in rotating detonation engine chamber," Proceedings of the Combustion Institute, 2016, pp. - .

133ahmoudi, Y., Karimi, N., Deiterding, R., and Emami, S., "Hydrodynamic Instabilities in Gaseous Detonations: Comparison of Euler, Navier-Stokes, and Large-Eddy Simulation," Journal of Propulsion and Power, Vol. 30, No. 2, 2014, pp. 384-396.

${ }^{14}$ Koo, H., Donde, P., and Raman, V., "A Quadrature-based LES/Transported Probability Density Function Approach for Modeling Supersonic Combustion," Proceedings of the Combustion Institute, Vol. 33, 2011, pp. 2203-2210.

$\checkmark{ }^{15}$ Koo, H., Donde, P., and Raman, V., "LES-based Eulerian PDF approach for the simulation of scramjet combustors," Proceedings of the Combustion Institute, Vol. 34, No. 2, 2013, pp. 2093-2100.

$\checkmark{ }^{16}$ Donde, P., Koo, H., and Raman, V., "A multivariate quadrature based moment method for LES based modeling of supersonic combustion," Journal of Computational Physics, Vol. 231, No. 17, 2012, pp. 5805-5821.

${ }^{17}$ Fiévet, R., Koo, H., and Raman, V., "Numerical simulation of a scramjet isolator with thermodynamic nonequilibrium," 22nd AIAA Computational Fluid Dynamics Conference, 22-26th June 2015, Dallas, TX, USA, No. AIAA-2015-3418, 2015.

${ }^{18}$ Fiévet, R., Voelkel, S., Koo, H., Varghese, P., and Raman, V., "Effect of Thermal Nonequilibrium on Ignition in Scramjet Combustors," Accepted for the 36rd Symposium (International) on Combustion.

${ }^{19}$ Mueller, M., Kim, T., Yetter, R., and Dryer, F., "Flow reactor studies and kinetic modeling of the H-2/O-2 reaction," International Journal of Chemical Kinetics, Vol. 31, No. 2, FEB 1999, pp. 113-125.

${ }^{20}$ Kee, R. J., Rupley, F. M., and Miller, J. A., Chemkin-II: A Fortran Chemical Kinetics Package for the Analysis of Gas Phase Chemical Kinetics, 1989, Sandia Report.

${ }^{21}$ Mcbride, B., Gordon, S., and Reno, M., "Coefficients for calculating thermodynamic and transport properties of individual species," NASA Technical Report NASA-TM-4513, NASA Lewis Research Center, 1993.

22 Jiang, G.-S. and Peng, D., "Weighted ENO Schemes for Hamilton-Jacobi Equations," SIAM Journal of Scientific Computing, Vol. 21, No. 6, 2000, pp. 2126-2143.

${ }^{23}$ Herrmann, M., Blanquart, G., and Raman, V., "A Bounded QUICK Scheme for preserving scalar bounds in large-eddy simulations," AIAA Journal, Vol. 44, No. 12, 2006, pp. 2879-2880.

${ }^{24}$ Powers, J. and Paolucci, S., "Accurate spatial resolution estimates for reactive supersonic flow with detailed chemistry," AIAA JOURNAL, Vol. 43, No. 5, MAY 2005, pp. 1088-1099, AIAA 43rd Aerospace Sciences Meeting and Exhibit, Reno, NV, JAN 10-13, 2005.

${ }^{25}$ Oran, E., Simulations of Gas Phase Detonations: Introduction of an Induction Parameter Model, NRL memorandum report, Naval Research Laboratory, 1980.

${ }^{26}$ Sichel, M., Tonello, N. A., Oran, E. S., , and Jones, D. A., "A TwoStep Kinetics Model for Numerical Simulation of Explosions and Detonations in H2O2 Mixtures," Proceedings of the Royal Society of London, Series A, Vol. 458, 2002 , pp. 49-82.

${ }^{27}$ Clifford, L., Milne, A., Turnyi, T., and Boulton, D., "An Induction Parameter Model for Shock-Induced Hydrogen Combustion Simulations," Combustion and Flame, Vol. 113, No. 12, 1998, pp. 106 - 118.

${ }^{28}$ Schwer, D. and Kailasanath, K., "Numerical investigation of the physics of rotating-detonation-engines," Proceedings of the Combustion Institute, Vol. 33, No. 2, 2011, pp. 2195 - 2202.

$\checkmark{ }^{29}$ Nordeen, C. A., Schwer, D., Schauer, F., Hoke, J., Barber, T., and Cetegen, B. M., "Role of inlet reactant mixedness on the thermodynamic performance of a rotating detonation engine," SHOCK WAVES, Vol. 26, No. 4, JUL 2016, pp. 417-428. 